\title{
CLSM and TIRF images from lignocellulosic materials: garlic skin and agave fibers study.
}

Josué Hernández-Varela ${ }^{1}$, José Chanona-Pérez ${ }^{2}$, Héctor Calderón Benavides ${ }^{3}$, Susana Gallegos Cerda ${ }^{4}$, Lizbeth Gonzalez Victoriano ${ }^{5}$, María de Jesús Perea Flores ${ }^{6}$, Maximiliano Campos López ${ }^{5}$, Liliana Edith Rojas Candelas ${ }^{7}$ and Benjamín Arredondo Tamayo ${ }^{5}$

${ }^{1}$ Laboratorio de micro y nanobiotecnología, Departamento de Ingeniería Bioquímica, Escuela Nacional de Ciencias Biológicas, Instituto Politécnico Nacional, Mexico city, Mexico., Ciudad de mexico, Distrito Federal, Mexico, ${ }^{2}$ Laboratorio de micro y nanobiotecnología, Departamento de Ingeniería Bioquímica, Escuela Nacional de Ciencias Biológicas, Instituto Politécnico Nacional, Mexico city, Mexico., Mexico City, Distrito Federal, Mexico, ${ }^{3}$ Departamento de Física, Escuela Superior de Física y Matemática, Instituto Politécnico Nacional, Mexico city, Mexico, Distrito Federal, Mexico, ${ }^{4}$ Laboratorio de micro y nanobiotecnología, Departamento de Ingeniería Bioquímica, Escuela Nacional de Ciencias Biológicas, Instituto Politécnico Nacional, Mexico city, Mexico., Mexico, Distrito Federal, Mexico, ${ }^{5}$ Laboratorio de micro y nanobiotecnología, Departamento de Ingeniería Bioquímica, Escuela Nacional de Ciencias Biológicas, Instituto Politécnico Nacional, Mexico city, Mexico., Distrito Federal, Mexico, ${ }^{6}$ Centro de Nanociencias y Micro y Nanotecnologías, Instituto Politécnico Nacional, Mexico city, Mexico, Distrito Federal, Mexico, ${ }^{7}$ IPN, Mexico, Distrito Federal, Mexico

\section{ABSTRACT}

Fluorescence techniques have been widely used by scientists to reveal valuable information from biological samples, but in food science, small progress is known due to the complexity of the samples. In this study, two different biological samples, garlic skin (GS) and agave fibers (AF), were used to evaluate the techniques of confocal laser scanning microscopy (CLSM) and total internal reflection fluorescence (TIRF) microscopy, to obtain valuable information on the fiber size of the samples. A compositional characterization with calcofluor white in CLSM was achieved, but a superficial characterization of the samples with TIRF was made, evidencing fiber sizes of $398.67 \pm 48.47 \mathrm{~nm}$ and $677.38 \pm 76.88 \mathrm{~nm}$ for GS and $\mathrm{AF}$, respectively. This work reveals that only an untreated sample can be used with the two techniques in the same microscope. In addition, it is possible to characterize the sample only using a spatial field of research and which valuable information about the structure of the material is found. This work provides the opportunity to use advanced fluorescence techniques for elucidation of structures shortly before studied with these techniques.

\section{MAIN TEXT}

Fluorescence microscopy is known as a fundamental set of methods for visualizing stained structures in the system under various conditions [1]. Fluorescence microscopy is generally used in the life sciences, but some studies reveal that new techniques could achieve greater characterization of the material without being a living system [2]. Normally, a biological sample can be stained with a fluorescent 
molecule, either synthetic or biological, and subsequently, a structure of interest is observed in more detail or definition [2,3]. Fluorescent molecules can be visualized based on two main factors: sample preparation and the targets used, but the resolution at which samples can be observed varies according to technique and may be limited by unfocused light capture in a focal plane or an evanescence plane [4,5]. The technique in which a focal plane is used to observe the fluorescence molecules in a sample is known as confocal laser scanning microscopy (CLSM) and this is one of the most useful techniques in the area of life sciences. CLSM has a wide range of fluorophores that can be used in many different ways to achieve a detailed sample in the biological field [3], but the resolution of the technique is lower compared to more innovative techniques since the focal plane and targets can only reach the resolution based on the Abbe diffraction limit $(r=0.511 / \mathrm{NA})[1,6]$. For example, using a 100X/1.46 lens and an excitation wavelength of $405 \mathrm{~nm}$, Abbe's law can only achieve a minimum resolution (r) of $140 \mathrm{~nm}$ at best when the sample is perfectly prepared and without crossing the emission wavelength. Are. On the other hand, a technique in which resolution is achieved by an evanescence plane is known as total internal reflection fluorescence microscopy (TIRF) and makes use of specific optics to produce illumination light only in the range of 50$100 \mathrm{~nm}$ at the interface. of the slide [1,7]. This technique drastically reduces the light bulb and improves the ability to detect fluorescent molecules only on the surface of the sample [8]. The main advantage of TIRF over CLSM is low light intensity and high spatial resolution, factors that make TIRF a key technique in obtaining topographic images. For this study, two different biological samples, garlic skin (GS) and agave fibers (AF), were used to evaluate CLSM and TIRF techniques and characterized the sample only using a spatial field of research.

\section{MATERIALS AND METHODS}

For imaging, an inverted confocal laser scanning microscope (LSM 880, Carl Zeiss, Germany) was used to observe the cellulose microfiber of GS and AF. Samples were stained with a white calcofluor solution (fluorescent brightener 28 F3543, Sigma-Aldrich, USA). For CLSM and TIRF, labeled samples were observed using a Zeiss a-Plan Apochromat M27 oil immersion lens of $100 \times 1.46$ for Elyra. For CLSM, samples were excited with a $405 \mathrm{~nm}$ laser using a 410-523 nm detection window. For TIRF, imaging was performed on an Andor1 Axio observed camera (Andor Technology, Belfast, Ireland) using a $405 \mathrm{~nm}$ Elyra laser for excitation, a bandpass filter with 420-480 + $750 \mathrm{~nm}$. For TIRF imaging, samples were observed with epi-fluorescence mode to adjust focus on the surface. Then, the TIRF mode with angle variation was chosen until the surface became visible (around $65^{\circ}$ ) with a value of $<200 \mathrm{~nm}$ of penetration. An area of $1280 \times 1280$ pixels was selected with a collimator gain of 600 and frame exposure times of $106 \mathrm{~ms}$. For a better TIRF structure, a small area of 500 x 500 with the same parameters was observed. For CLSM and TIRF, the same $\mathrm{x}-\mathrm{y}-\mathrm{z}$ scan area was selected for better comparison.

\section{RESULTS AND DISCUSSION}

Figure 1 shows the CLSM images obtained from GS (Fig. 1a) and AF (Fig. 1b). GS presented an organized structure in laminar form with a characteristic form of the cell wall; thus, AF presented a more fibrillar, linear, and compact composition [9]. In the first analysis, Fig. 1a presented more valleys in the 
surrounding formation of cell walls, since studies reveal a characteristic formation of sclerenchyma fibers that are formed by cellulose in the samples. In addition, Fig. 1b presented a linear form as fibers in which the septa cells are the surrounding structure without the blue fluorescence of the cellulose because this structure is rich in lignin [9].

A significant disclaimer should be made, both Fig. 1a and $1 \mathrm{~b}$ presented an oversaturated fluorescence image due to the focal point chosen for these images. This behavior makes it difficult for these images to observe a detailed topography of the sample.

However, TIRF microscopy is used in the same area as CLSM images. Figure 2 shows the TIRF images obtained from GS (Fig. 2a and 2b) and AF (Fig. 2c and 2d). Changes in the surface are observed with this technique, since the angle of incidence is minimal and the evanescent waves generated by the TIRF illumination decay exponentially, causing a small intensity up to $~ 100 \mathrm{~nm}$ of depth.

This behavior allows to reduce the phototoxic effects, the total light in the sample is dosed to a minimum and the fluorescence out of focus is eliminated, which gives a much higher signal-to-noise ratio $[1,4]$. It is important to note that using TIRF images it is possible to measure the width of the cellulose microfiber packages from a very easy measurement tool, which is impossible using CLSM images. In both cases, GS and AF showed structures with sizes of $398.67 \pm 48.47 \mathrm{~nm}$ (Fig. 2b) and $677.38 \pm 76.88$ nm (Fig. 2d), respectively.

\section{CONCLUSIONS}

In general, TIRF allows you to observe details in a sample in which the fluorophores are more prominent without compromising the resolution or character of the sample. In this case, TIRF is used as a surface characterization technique, producing a good characterization on a smaller scale, but in the same spatial field as CLSM. The main idea of these well-founded results is the use of TIRF as a novel technique not only in biological material but also in films or composites in which one or more fluorophores could be used.

\section{Acknowledgments}

The authors thank the financial support of COFAA from the Instituto Politécnico Nacional, SIP project (Clave: 2019) and the multidisciplinary project (Clave: 20210065) for the support received. 


\section{Confocal Laser Scanning Microscopy} (CLSM)

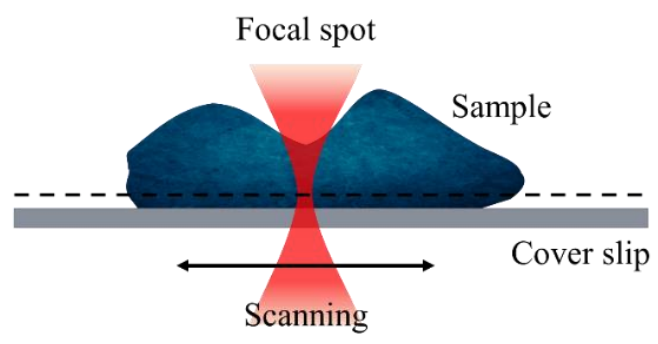

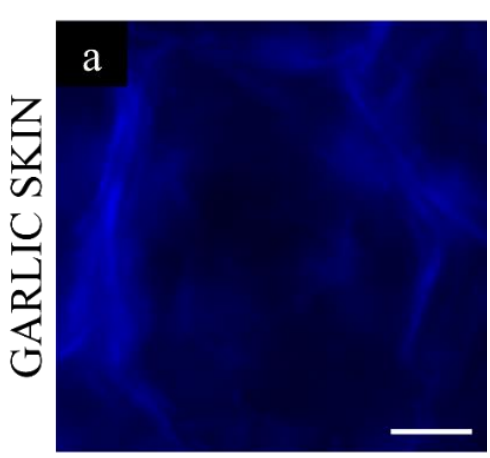

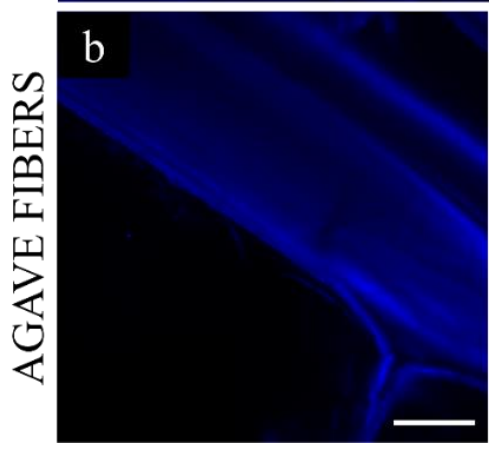

Figure 1. Schematic representation of confocal laser scanning microscopy (CLSM) of garlic skin (a) and agave fibers (b) dyed with calcofluor (blue). Scale bar of $10 \mathrm{um}$.

Total Internal Reflection Fluorescence

(TIRF) Microscopy

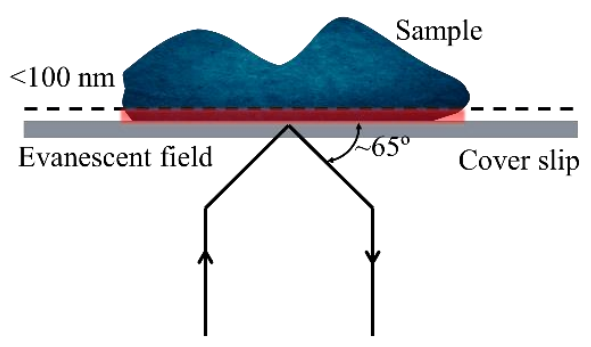

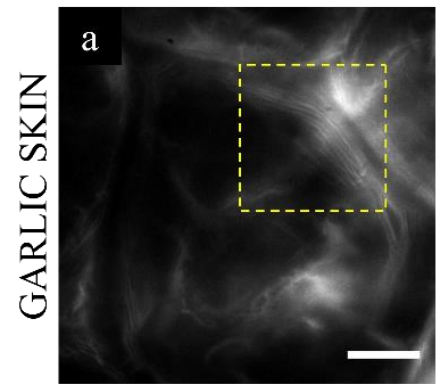
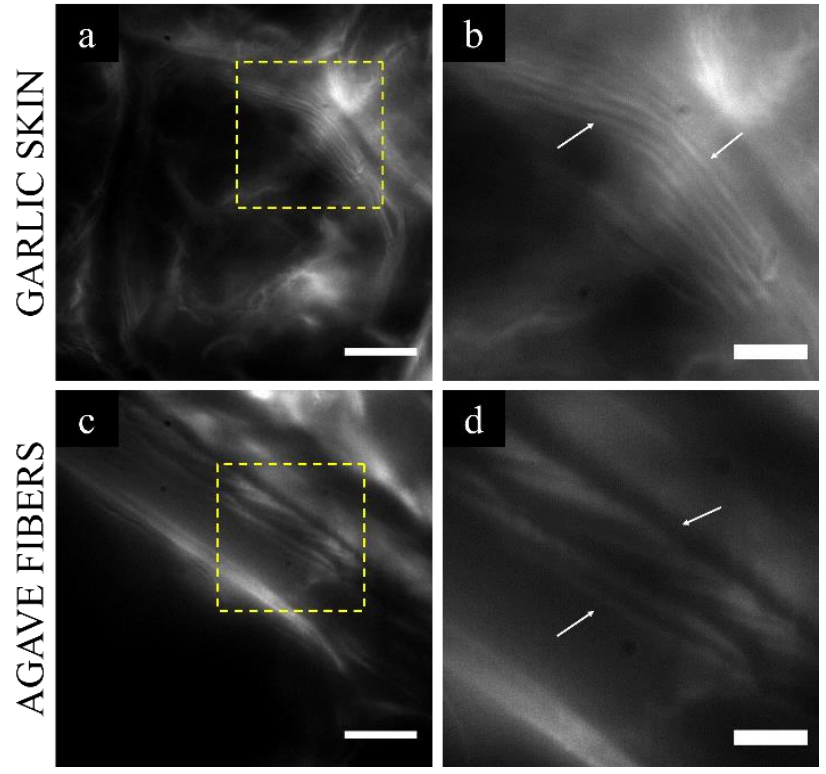

Figure 2. Schematic representation of total internal reflection fluorescence microscopy (TIRF) for garlic skin (a) and agave fibers (b) stained with calcofluor target and using a reflection angle of $65^{\circ}$. Scale bar of $10 \mathrm{um}$. Square inserts with yellow dashes are displayed on a scale of $4 \mathrm{um}(\mathrm{b}, \mathrm{d})$. 


\section{References}

[1] J.S. Danial, Y. Aguib, M.H. Yacoub, Advanced fluorescence microscopy techniques for the life sciences, Glob. Cardiol. Sci. Pract. 2016 (2016). https://doi.org/10.21542/gcsp.2016.16.

[2] J. Tang, J. Ren, K.Y. Han, Fluorescence imaging with tailored light, Nanophotonics. (2019). https://doi.org/10.1515/nanoph-2019-0227.

[3] O.O. Onipe, D. Beswa, A.I.O. Jideani, Confocal laser scanning microscopy and image analysis for elucidating crumb and crust microstructure of bran-enriched South African fried dough and batter, Foods. 9 (2020) 1-22. https://doi.org/10.3390/foods9050605.

[4] A.L. Mattheyses, S.M. Simon, J.Z. Rappoport, Imaging with total internal reflection fluorescence microscopy for the cell biologist, J. Cell Sci. 123 (2010) 3621-3628. https://doi.org/10.1242/jcs.056218.

[5] J. Liesche, I. Ziomkiewicz, A. Schulz, Super-resolution imaging with Pontamine Fast Scarlet 4BS enables direct visualization of cellulose orientation and cell connection architecture in onion epidermis cells., BMC Plant Biol. 13 (2013) 226.

[6] I.M. Khater, I.R. Nabi, G. Hamarneh, A Review of Super-Resolution Single-Molecule Localization Microscopy Cluster Analysis and Quantification Methods, Patterns. 1 (2020) 100038. https://doi.org/10.1016/j.patter.2020.100038.

[7] B. Huang, S.A. Jones, B. Brandenburg, X. Zhuang, Whole-cell 3D STORM reveals interactions between cellular structures with nanometer-scale resolution, Nat. Methods. 5 (2008) 1047-1052. https://doi.org/10.1038/nmeth.1274.

[8] C.L. Asbury, Data analysis for total internal reflection fluorescence microscopy, Cold Spring Harb. Protoc. 2016 (2016) 471-473. https://doi.org/10.1101/pdb.prot085571.

[9] J.D. Hernández-Varela, J.J. Chanona-Pérez, H.A. Calderón Benavides, F. Cervantes Sodi, M. Vicente-Flores, Effect of ball milling on cellulose nanoparticles structure obtained from garlic and agave waste, Carbohydr. Polym. (2021). https://doi.org/10.1016/j.carbpol.2020.117347. 